\title{
MACHINE LEARNING VERSUS HUMAN-DEVELOPED ALGORITHMS IN IMAGE ANALYSIS OF MICROSTRUCTURES
}

doi: 10.2478/cqpi-2019-0056

Date of submission of the article to the Editor: 08/04/2019

Date of acceptance of the article by the Editor: 22/05/2019

Adam Piwowarczyk ${ }^{1}$ - orcid id: 0000-0001-6006-1065

Leszek Wojnar ${ }^{1}$ - orcid id: 0000-0001-8786-3535

${ }^{1}$ Cracow University of Technology, Cracow, Poland

Abstract: Automatic image analysis is nowadays a standard method in quality control of metallic materials, especially in grain size, graphite shape and non-metallic content evaluation. Automatically prepared solutions, based on machine learning, constitute an effective and sufficiently precise tool for classification. Human-developed algorithms, on the other hand, require much more experience in preparation, but allow better control of factors affecting the final result. Both attempts were described and compared.

Keywords: image analysis, object detection, neural networks, machine learning

\section{INTRODUCTION}

After approximately five decades of development in image analysis techniques they became a tool for everyday use. Optical character recognition for automatic conversion of bitmaps into editable text or automatic identification of car license plates, industrial quality control, traffic control, face recognition and other biometry applications, medical diagnostics and reading barcodes or QR codes are just selected examples of their application.

Various software packages offer a wide spectrum of image transformations allowing improvement of image quality using brightness and contrast as well as geometrical correction, filters for noise removal or enhancement of some features visible in an image, frequency operations, mathematical morphology transformations being in fact advanced filters, numerous techniques for object recognition and quantitative evaluation. All these techniques are thoroughly explained in numerous textbooks. However, there is always a serious problem in elaboration of suitable algorithms. The first book devoted mainly to development of working solutions was published in 1999 (Wojnar, 1999) and till now we observe a lack of similar, advanced works.

During the last years a successful solution of the above mentioned problems was found. The use of deep learning and neural networks gives us great opportunities to become independent from the problems of proper algorithm design and allows effective recognizing of objects in images. However, both attempts have their advantages and constraints which are described later in this paper. 


\section{CLASSICAL IMAGE ANALYSIS}

The problems of classical image analysis are presented on the example of detection of grain boundaries in sintered $\mathrm{CeO} 2$ observed in scanning electron microscope (fig. 1a). Location of grain boundaries is well visible for human eyes, but difficult for recognition using image analysis techniques. Any attempt to do it using single transformation fails. One of the best results, obtained using entropy automatic thresholding is shown in fig, 1b. Discontinuity of grain boundary lines with missing long segments disables any further analysis. In order to solve this problem two algorithms, called A nad B respectively, were elaborated and applied.

Algorithm A

- Edge detection using Prewitt filter

- Median filtering for noise reduction

- Erosion in order to get possibly continuous grain boundaries

- Closing holes

- Detection of missing boundaries using watershed segmentation

- SKIZ (skeleton by influence zone) in order to get single-pixel grain boundaries.

Final result of detection using this algorithm is presented in fig.1c. Lack of numerous grain boundaries easily detected by a human eye is clearly visible. Most of the errors can be avoided if one uses a more complex algorithm:

Algorithm B

- Edge detection using Prewitt filter

- White top-hat transformation in order to detect bright pixels which have, surprisingly, greater density at grain boundaries

- Black top-hat in order to detect the darkest regions in the initial image which also have greater density at grain boundaries

- Logical AND of the three images listed above, which summarizes all the darkest points

- Cleaning the noisy image using median filter

- Erosion which improves continuity of boundary lines

- Dilation which corrects irregular shape of grains

- Detection of the missing boundaries using watershed segmentation

- SKIZ as a final step of image processing.

Final result of detection using algorithm $B$ is shown in fig. 1d. There are still some detection errors visible, but the majority of grain boundaries is well detected and quantitative evaluation of the grainy structure can be performed without any risk of significant errors.

Possibly part of the transformations listed in algorithms $A$ and $B$ can be not clear for the readers not being specialists in image analysis, but description of these algorithms was added just in order to illustrate the complexity and problems in preparation of image processing algorithms. Detailed explanation of both algorithms as well as individual transformations can be found in a book (Wojnar, 1999).

This example illustrates complexity of image processing algorithms. They can be significantly more complicated in the case of unevenly illuminated structures observed in light microscopes which requires shade correction, images partly out of focus or during analysis of highly nonhomogeneous structures. To make thing worse, every, 
even very effective, algorithm can be applied only in analysis of a relatively narrow group of structures.

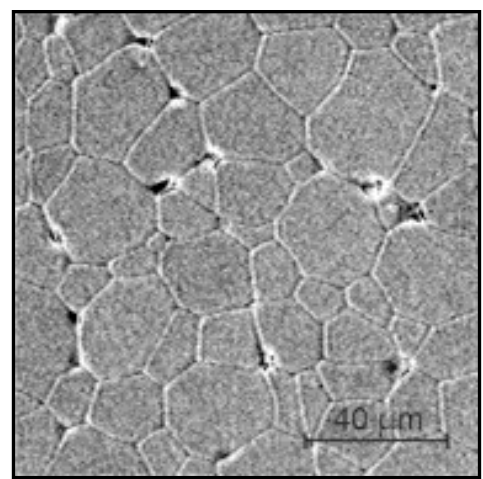

a

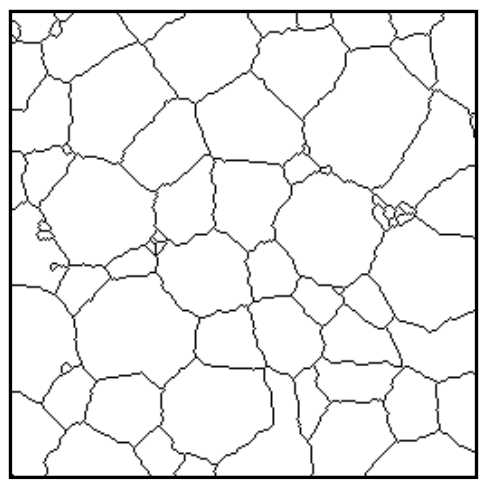

C

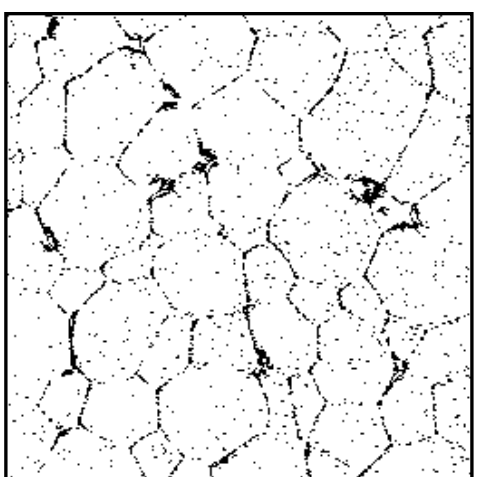

b

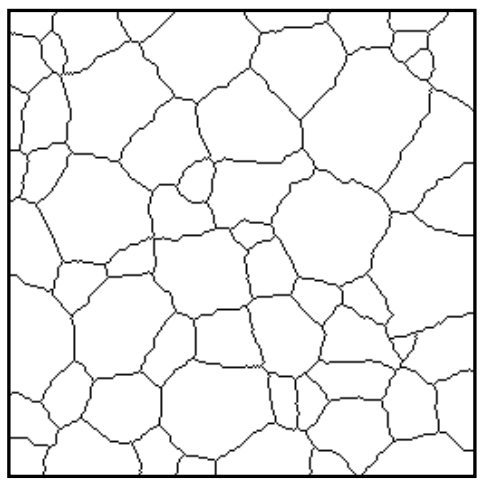

d

Fig. 1. Detection of grain boundaries in a $\mathrm{CeO}_{2}$ ceramic observed in SEM: initial image (a) and binary images after: entropy threshold (b), algorithm A (c) and algorithm B (d)

Classical algorithms can be elegant and effective, but their preparation cannot be completed without deep knowledge of metallography techniques and thorough understanding of image processing transformations. Additionally, some imaginary skills are recommended for solving new and complex problems. Consequently, preparation and validation of new algorithms is usually time consuming and requires a lot of experience of the operator.

In order to run any algorithm appropriate computer system and programming language is necessary. Numerous solutions are available, part of them are Open Source software. Every system has its own advantages and drawbacks. Therefore one cannot recommend any software solution as an ideal choice.

\section{MACHINE LEARNING}

Neural networks are a main tool for machine learning. They can be interpreted as a versatile and scalable mathematical model whose task is to process information. Neural networks can be applied to large and complex tasks such as image analysis (Geron, 2018, Zocca et al., 2017).

Neural networks can be significantly differentiated due to network architecture, learning method and activation function. An example of a neural network suitable for image analysis is a group of convolutional networks (CNN - convolutional neural network). Their results exceed human abilities to interpret images. This happens thanks to the continuously growing computing power and a large amount of available training data. 
The use of CNN networks allows not only for image analysis but also automated film classification systems, speech recognition and natural language processing (Geron, 2018).

Machine learning can be useful in various tasks that require analysis and interpretation of complex data sets. Machine learning process can be organized in three modes: supervised learning, unsupervised learning and reinforcement learning. Supervised learning uses a set of labeled data to organize similar, unidentified data. Unsupervised learning does not assign data in advance, but the algorithm can draw conclusions by itself. Reinforcement learning allows for correlating immediate actions with their delayed results. It can be very useful in real life applications and the potential of this way of learning is demonstrated in teaching the machine to play games (Zocca et al., 2017). Over the last few years, many publications are connected with application of neural networks and machine learning in searching for objects in images representing microstructures and macrostructures, like cracks in the roads or bridges (Brian et al., 2015, Chowdhury et al., 2016, Prasanna et al., 2016, Shi et al., 2016).

An interesting technique for detection of objects in an image, based on neural networks, was named YOLO (You Only Look Once). It allows detection of elements in the image after defining them prior to the final analysis (Redmon et al., 2015).

Machine learning constitutes a very promising tool in image analysis. However, usually it requires a lot of training examples and the methodology is still far from being complete. Nevertheless, machine learning seems to be the most promising tool which can face the growing number of problems connected with automatic image acquisition and further analysis.

\section{DISCUSSION}

This paper does not contain examples of machine learning applications in materials engineering, especially metallography. This is due to the necessity of collecting large data sets for training purposes. The images should be of good quality and represent representative differentiation of microstructures. There are some promising examples, but still not ready for publication.

In spite of this one has enough data to compare image analysis of microstructures based on classical algorithms and solutions obtained using machine learning. Some items from this comparison are shown in table 1. It seems to be clear that in the coming close future machine learning will become more and more significant and frequently used tool in image analysis. So, our goal should be development of appropriate standard procedures as well as tools for evaluation of the results.

At the moment machine learning seems to be unbeatable in automatic classification or even quantitative evaluation of materials microstructures. Possibly some hybrid methods will be the most successful ones. They should combine some classical image processing in order to enhance or pre-process the initial images and machine learning solutions for preliminary selection and and final classification.

Possible a niche for classical algorithmic analysis could be low level research where one searches for explanation of the very basic rules of material behavior in new environments or loadings. Classical algorithms based on some scientific hypotheses can be helpful in their experimental verification. 
Table 1

Comparison of analysis based on machine learning and human developed algorithms

\begin{tabular}{|l|l|l|}
\hline \multicolumn{1}{|c|}{ Analysed feature } & $\begin{array}{c}\text { Analysis based on machine } \\
\text { learning }\end{array}$ & \multicolumn{1}{|c|}{$\begin{array}{c}\text { Human-developed } \\
\text { algorithms }\end{array}$} \\
\hline $\begin{array}{l}\text { data necessary to develop } \\
\text { a complete application }\end{array}$ & $\begin{array}{l}\text { large data set necessary for } \\
\text { training }\end{array}$ & $\begin{array}{l}\text { small data set can be } \\
\text { sufficient for development of } \\
\text { the algorithm }\end{array}$ \\
\hline required experience of the & $\begin{array}{l}\text { medium experience in } \\
\text { machine learning should be } \\
\text { sufficient }\end{array}$ & $\begin{array}{l}\text { extensive experience in } \\
\text { image analysis and } \\
\text { metallography is required }\end{array}$ \\
\hline speed of analysis & can be high & can be high \\
\hline required image quality & $\begin{array}{l}\text { depends on the training data; } \\
\text { usually high quality of initial } \\
\text { images is necessary }\end{array}$ & $\begin{array}{l}\text { depends on the experience } \\
\text { of the person preparing } \\
\text { algorithms; can accept } \\
\text { medium quality images }\end{array}$ \\
\hline potential field of application & $\begin{array}{l}\text { routine quality control and } \\
\text { large data sets }\end{array}$ & $\begin{array}{l}\text { research and routine quality } \\
\text { control }\end{array}$ \\
\hline time necessary to prepare & $\begin{array}{l}\text { depends on the problem } \\
\text { analysed; usually relatively } \\
\text { long }\end{array}$ & $\begin{array}{l}\text { depends on the problem } \\
\text { analysed; can be short } \\
\text { mainly in the case of semi- } \\
\text { automatic analysis }\end{array}$ \\
\hline main advantages & $\begin{array}{l}\text { ease of use of the final } \\
\text { solution }\end{array}$ & $\begin{array}{l}\text { possible successful } \\
\text { application in advanced } \\
\text { research }\end{array}$ \\
\hline main constraints & $\begin{array}{l}\text { results are a function of size } \\
\text { and quality of the training set } \\
\text { as well as of training method }\end{array}$ & $\begin{array}{l}\text { methods usually applicable } \\
\text { to a limited set of images, } \\
\text { strongly related to the } \\
\text { operator's experience }\end{array}$ \\
\hline
\end{tabular}

Source: data prepared by the authors

\section{REFERENCES}

Brian L. DeCost, Elizabeth A. Holm, 2015. A computer vision approach for automated analysis and classification of microstructural image data, Computational Materials Science, 110, 126133, DOI: 10.1016/j.commatsci.2015.08.011

Chowdhury A., Kautz E., Yener B., Lewis D., 2016. Image driven machine learning methods for microstructure recognition, Computational Materials Science, 123, 176-187, DOI: 10.1016/j.commatsci.2016.05.034.

Geron A., 2018. Uczenie maszynowe z użyciem Scikit-Learn i TensorFlow, Helion SA, Gliwice Poland [in Polish]

Prasanna P., Dana K. J., Gucunski N., Basily B. B., Hung M. La, Lim R. S., Parvardeh H., 2014. Automated Crack Detection on Concrete Bridges, IEEE Transactions on Automation Science and Engineering, 13, 591-599,

DOI: 10.1109/TASE.2014.2354314

Redmon J., Divvala S., Girshick R., Farhadi A., 2015. You Only Look Once. Unified, Real-Time Object Detection, 2016 IEEE Conference on Computer Vision and Pattern Recognition (CVPR), 779-788, DOI: 10.1109/CVPR.2016.91.

Shi Y., Cui L., Qi Z., Meng F., Chen Z., 2016. Automatic Road Crack Detection Using Random Structured Forests, IEEE Transactions on Intelligent Transportation Systems, 17, 34343445, DOI: 10.1109/TITS.2016.2552248

Wojnar, L., 1999. Image analysis. Applications in materials engineering, CRC Press, Boca Raton, USA.

Zocca V., Spacagna G., Slater D., Roelants P., 2017. Deep learning. Uczenie głębokie z językiem Python. Sztuczna inteligencja i sieci neuronowe, Helion SA, Gliwice Poland [in Polish] 